\title{
Study of Local Irritant Action of a Prototype Drug Based on Esters of Fatty Acids
}

\author{
Ksenia Smirnova*, Olga Larionova, and Boris Drevko \\ Federal State-Owned Publicly-Funded Institution of Higher Education Saratov State Agrarian \\ University named after N.I. Vavilov, Saratov, 1 Teatralnaya Ploshchad, 410012, Russian Federation
}

\begin{abstract}
In this article, we have presented studies of the local irritating effect of a prototype wound-healing preparation based on water-based fatty acid esters with allantoin. The study of local irritant action included use of extreme dilutions of the drug for intradermal and local use in the induction and provocative phases. The assessment was carried out in points, in the experimental group it was on average 1 point, in control animals on average it was also equal to 1 . These studies indicate that the composition has a mild skin reaction of sensitized guinea pigs and does not cause an allergic reaction.
\end{abstract}

\section{Introduction}

Among the raw materials used in medicines and cosmetics, lipids occupy one of the main places. For a long time, the most readily available animal fats and vegetable oils, such as lard and fat, palm, olive and almond oils, have been used [1]. They consist of triacylglycerides of higher saturated and unsaturated fatty acids. This chemical composition largely determines the ease of their penetration into the hair follicle and the upper layers of the epidermis. They are the most suitable bases for nourishing and, to a lesser extent, protective creams $[2,3]$.

A negative property of lipids is their ability to oxidize and rancid, associated with the unsaturated nature of triacylglycerides of fatty acids. The products of these processes aldehydes, ketones and fatty acids - irritate the skin and mucous membranes. Also, as a rule, in addition to plant and natural components, the composition of drugs includes various excipients that can cause various allergic reactions [4].

In this regard, the study of local irritant and anti-allergic properties are of paramount importance.

\section{Methods}

For the study, we chose a prototype of a wound-healing drug Sample 6, which we developed, consisting of a complex of fatty acid esters $13 \%$, allantoin $10 \%$, benzyl alcohol

\footnotetext{
* Corresponding author: smirnova.ku@mail.ru
} 
$1 \%$, dimethyl sulfoxide (DMSO) $10 \%$, carboxymethyl cellulose (CMC) $1 \%$, glycerol $5 \%$, talc $33 \%$, aloe juice $10 \%$, TWIN-80 $5 \%$ and water.

For the study, we took healthy young sexually mature male guinea pigs of the same line, weighing 300-400 g before the start of the experiment. To prepare the animals for testing, one day before the start of the study, the hair on the experimental skin areas was carefully cut. The animals were divided into 2 groups - experimental and control, 10 animals each.

Research began with the induction phase. Extreme dilutions were tested that did not cause more than very weak erythema, and did not have an overall adverse effect on the animals.

To prevent potential bias in the results, the effect of administering Freund's Complete Adjuvant (FCA) in combination with the test material during the main test was considered.

Each animal received paired intradermal injections in a volume of $0.1 \mathrm{ml}$ into the clipped skin areas (A, B, and C).

A - A mixture of Freund's complete adjuvant with a solvent in a 50/50 ratio.

B - Test sample; control animals were injected only with the vehicle.

C - Test sample at the concentration selected for the area, emulsified with complete Freund's adjuvant in a 50/50 ratio and solvent (50\%); control animals received only the emulsion of the control solution with adjuvant.

Seven days after the intradermal induction phase, the local induction phase was started. For this, skin applications of the test material were applied to the injection areas. For applications, a hygroscopic gauze pad of $8 \mathrm{~cm} 2$ was impregnated with the test sample, which was applied to the clipped skin areas and fixed with an occlusive dressing around the animal's body. We used freshly prepared solutions of maximum concentration. The dressing was removed after 48 hours.

In the control group of animals, the actions were identical, however, only the control solution was used.

14 days after the end of the local induction phase, the provocative phase was started for all experimental and control animals with the test material. For this, the test material was applied to the intact areas of the skin of each animal using a hygroscopic gauze pad. A fixing occlusive dressing was applied. The dressing was removed after 24 hours.

The surface of the areas was examined 24 and 48 hours after removing the dressing under daylight and artificial light.

The assessment of the condition of the experimental areas at each observation time interval for the presence of erythema and edema was carried out in accordance with Table 1 (according to the classification of Magnusson and Kligman).

Table 1. Skin reaction classification system.

\begin{tabular}{l|c}
\hline \multicolumn{1}{c|}{ Description of the response } & Points \\
\hline No visible changes & 0 \\
Discrete or focal erythema & 1 \\
Moderate to continuous erythema & 2 \\
Intense erythema and swelling & 3 \\
\hline
\end{tabular}

\section{Results}

When studying the local irritating effect after paired intradermal injection of substances in the corresponding areas of the skin in a volume of $0.1 \mathrm{ml}$ to each animal. After 24 hours, the results were recorded.

In the control group, at the injection area, there was a slight swelling at the injection area, which disappeared after 6-8 hours, the condition of the animals was stable, erythema was not observed (Figure 1). 


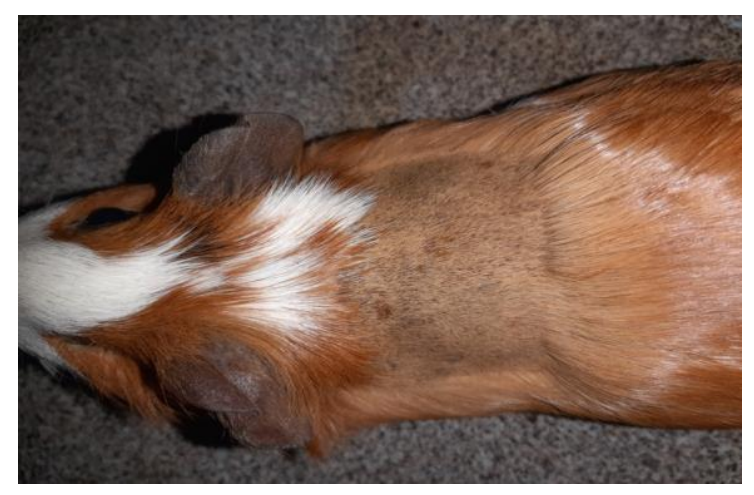

Fig. 1. Reaction at the injection area in the control group immediately after injection.

In the experimental group of animals, at the injection area of a mixture of Freund's complete adjuvant with the selected solvent in a 50/50 ratio, there was a slight swelling at the injection area, which disappeared after 6-8 hours, the condition of the animals was stable, and a slight erythema was observed.

At the injection area of the test sample with the solvent, there was a slight swelling at the injection area, which disappeared after 4-5 hours, the condition of the animals was slightly depressed, but after 1-2 hours it was stable, erythema was not observed (Figure 2).

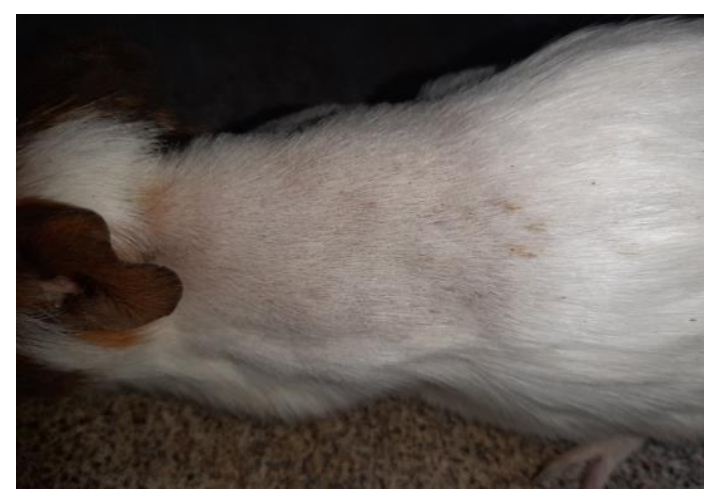

Fig. 2. Reaction at the injection area in the test group immediately after injection.

After introduction of the test sample at the concentration selected for the area, emulsified with complete Freund's adjuvant in a 50/50 ratio and a solvent (50\%), a slight swelling was observed at the injection area, which passed after 6-8 hours, the condition of the animals was slightly depressed, but after 2 -3 hours - stable, no erythema was observed (Figure 3). 


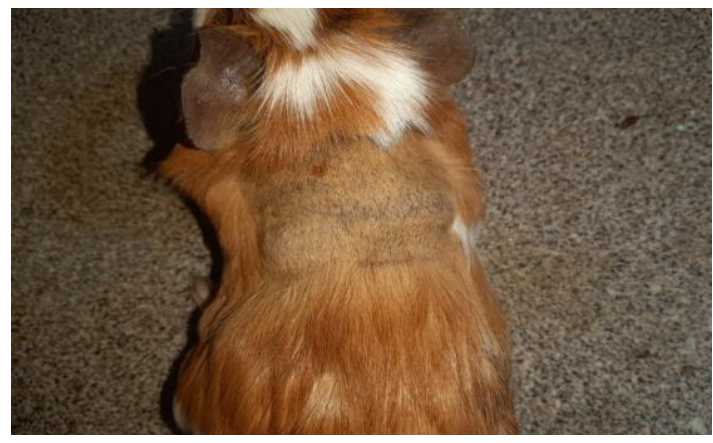

Fig. 3. Reaction at the injection area in the test group immediately after injection.

Seven days after the intradermal induction phase, skin applications of the test material were started on the injection areas, on the intra-scapular region of each animal, using hygroscopic gauze pads soaked in the sample with an area of $8 \mathrm{~cm} 2$. The surface of the skin areas of animals within 14 days after the application of skin applications of the test material in experimental and control animals was examined. At 24 hours after removing the dressing, moderate erythema was observed in all animals, including control ones (Figure 4).

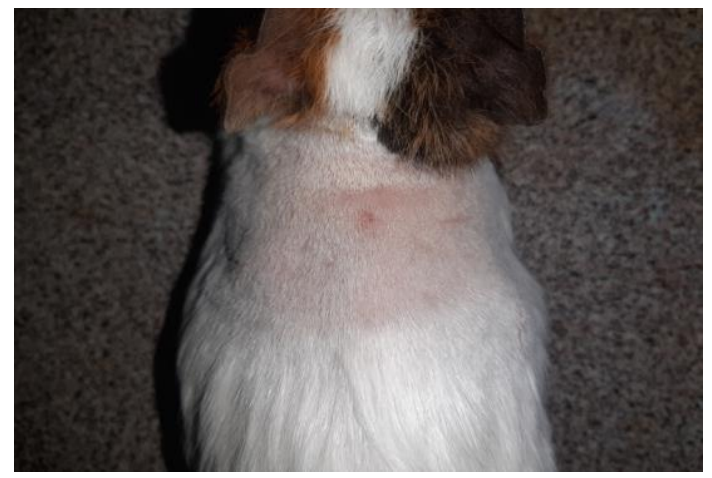

Fig. 4. Result in 24 hours after the skin application in the experimental group.

After 48 hours, a small discrete or focal erythema was observed in the experimental group of animals (Figure 5).

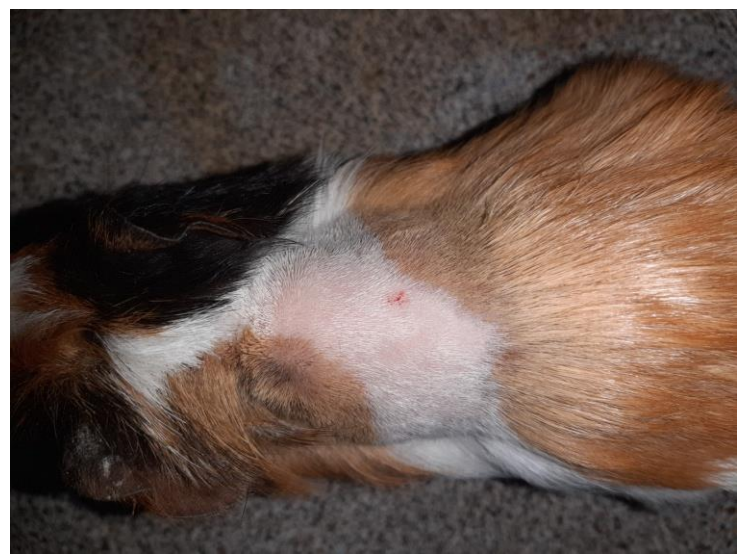

Fig. 5. Result in 48 hours after the cutaneous application. 
After 7 days, there was a slight swelling together with introduction in all groups of animals (Figure 6).

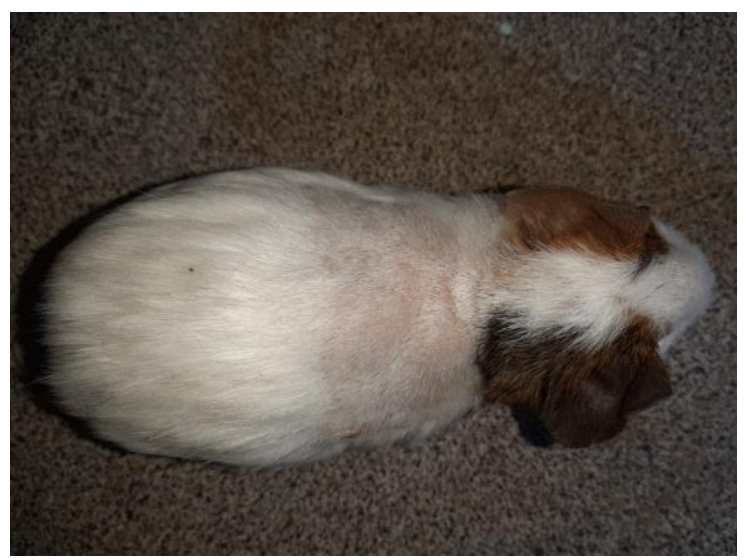

Fig. 6. The result on the 7th day after the skin application in the experimental group.

After 14 days, no changes were observed in all animals of the control and experimental groups (Figure 7).

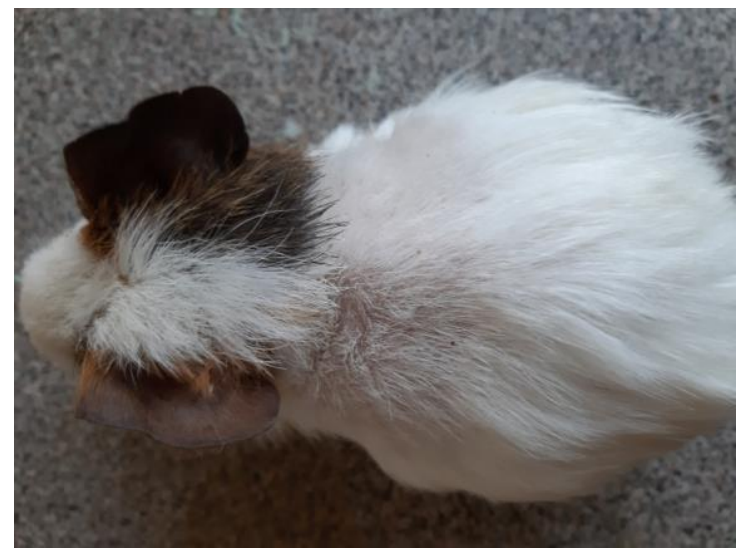

Fig. 7. Result on the 14th day after the cutaneous application.

When conducting the provocative phase in all experimental and control animals with the test material, the intact skin areas of the animals were assessed 24 and 48 hours after removing the dressing in daylight and artificial light in accordance with Table 1 (according to the classification of Magnusson and Kligman). Indicators on average were equal to 1 point in both study groups. There was little or no erythema.

\section{Discussion}

Skin wound healing is a dynamic process that involves complex cellular and extracellular mechanisms that can easily be threatened by chemicals, radiation or bacterial infections [5]. Therefore, it is imperative that any topical veterinary drug used does not interfere with wound healing, but protect the wound from infection. In addition, it shall provide a direct assessment of the wound, keep it moist and in contact with the wound surface even in the case of deep wounds. Moreover, it shall be well tolerated by animals $[6,7]$. 
Many ointments are currently available, but most of them do not meet all the desired criteria. Allergies and toxic effects are common adverse drug reactions [3, 8]. Adverse drug reactions, including allergic reactions, are one of the main causes of morbidity and mortality worldwide [9].

Esters of fatty acids hold a special place in the treatment of skin diseases and are widely used in medicine and veterinary medicine. They have anti-inflammatory, antiallergic, regenerating, bactericidal and antitumor properties $[4,10]$.

We carried out studies to study the local irritating effect of the prototype of the drug developed by us based on esters of fatty acids and allantoin on a water basis. This drug had mild reactions from the skin and, in this regard, it can be judged that this composition has no allergenic properties.

\section{Conclusion}

When conducting studies to determine the local irritating effect of the drug based on fatty acid esters after the induction phase, after 7 days, there was a slight swelling together with the introduction in all groups of animals. After 14 days, no changes were observed in all animals of the control and experimental groups. The condition of the animals was assessed as satisfactory. The average index of skin reaction when assessed according to the classification of Magnusson and Kligman in the experimental and control groups corresponded to 1 point.

After the provocative phase was carried out by the test material, the indices were on average 1 in all studied groups, as evidenced by little or no erythema.

According to the results of the study, it can be concluded that use of a permissive dose of the drug to sensitized guinea pigs causes a mild reaction on the part of the skin, which indicates the absence of allergenic properties in this composition.

\section{References}

1. A. V. Gubanov, YU. M. Postolov, A. N. Lisicyn, Sposob polucheniya smesi vysshih nenasyshchennyh zhirnyh kislot (2005)

2. O. Goertz, Eur. Sur. Res., 44 (2010)

3. Update in cosmetic dermatology (Berlin, Springer, 2013)

4. P. E. Somnet, J. A. Gazallino, J.Amer.Oil Chem.Soc, 68, 1 (1991)

5. GOST ISO 10993-10-2011 (2011)

6. YU. K. Abaev, Spravochnik hirurga. Rany i ranevaya infekciya (Rostov-na-Donu, Feniks, 2006)

7. L. A. Blatun, Consilium medicum, 1 (2007)

8. M. Tomljanovic-Veselski, J. Lipozencic, L. Lugovic, Coll Antropol, 31 (2007)

9. Bioactive dietary factors and plant extracts in dermatology (New York, Humana Press, 2013)

10. A. Kawabata, K. Miyatake, H. Inui, Àgr. and Biol. Chem, 54, 3 (1990) 